\title{
A Diagnostics Framework for Underground Power Cables Lifetime Estimation Under Uncertainty
}

\author{
Jose Ignacio Aizpurua, Member, IEEE, Brian G. Stewart, Member, IEEE, Stephen D. J. McArthur, Fellow, IEEE, \\ Nitin Jajware, Martin Kearns, Unai Garro, Eñaut Muxika and Mikel Mendicute Senior Member, IEEE
}

\begin{abstract}
Power cables are critical assets for the reliable operation of the grid. The cable lifetime is generally estimated from the conductor temperature and associated lifetime reduction. However, these tasks are intricate due to the complex physicsof-failure (PoF) degradation mechanism of the cable. This is further complicated with the different sources of uncertainty that affect the cable lifetime estimation. Generally, simplified or deterministic PoF models are adopted resulting in non-accurate decision-making under uncertainty. In contrast, the integration of uncertainties leads to a probabilistic decision-making process impacting directly on the flexibility to adopt decisions. Accordingly, this paper presents a novel cable lifetime estimation framework that connects data-driven probabilistic uncertainty models with PoF-based operation and degradation models through Bayesian state-estimation techniques. The framework estimates the cable health state and infers confidence intervals to aid decision-making under uncertainty. The proposed approach is validated with a case study with different configuration parameters and the effect of measurement errors on cable lifetime are evaluated with a sensitivity analysis. Results demonstrate that ambient temperature measurement errors influence more than load measurement errors, and the greater the cable conductor temperature the greater the influence of uncertainties on the lifetime estimate.

Index Terms-Condition monitoring, cable diagnostics, dynamic thermal rating, uncertainty, sensitivity.
\end{abstract}

\section{INTRODUCTION}

$\mathbf{P}$ OWER cables are critical assets for the reliable operation of the grid [1]. The unexpected cable failure can lead to lack of export capability or catastrophic failures depending on the system response and the associated circuit. The main ageing factors can be classified into [2]: thermal, electrical, mechanical and environmental causes leading to oxidation, partial discharges, electrical trees and space charges.

With the increase of available data sources across the power grid, there has been an increased interest in the implementation of Prognostics \& Health Management (PHM) strategies. Many PHM strategies focus on the development of data-driven solutions through machine learning methods which learn a model that represents the asset fault-to-failure progression taking

J. Aizpurua is with the Electronics \& Computing Department, Mondragon University, Arrasate, Spain and also with IKERBASQUE, Basque Foundation For Science, Bilbao, Spain (e-mail: jiaizpurua@mondragon.edu);

Brian G. Stewart \& Stephen D. J. McArthur are with the Inst. of Energy \& Environment, Univ. of Strathclyde, Glasgow, UK (e-mail: brian.stewart.100@strath.ac.uk; s.mcarthur@strath.ac.uk);

Nitin Jajware is with Bruce Power, Tiverton, Canada (e-mail: nitin.jajware@brucepower.com; Martin Kearns is with EDF-Energy, East Kilbride, UK (e-mail: martin.kearns@edf-energy.com);

U. Garro, E. Muxika \& M. Mendicute are with the Electronics \& Computing Department, Mondragon University, Arrasate, Spain (e-mail: \{ugarro, emuxika, mmendikute\}@mondragon.edu). into account influential parameters. However, the degradation modelling of power cables is a challenging task [1], and in many cases, the availability of run-to-failure data is limited.

\section{A. Related work}

1) Data-driven PHM solutions: Many data sources associated with power cables have been focused on diagnostic tests such as elongation at break [3], partial discharge [4], timereflectometry [5], insulation resistance [6] and polarization currents [7]. However, the limited availability of diagnostic tests has constrained the adoption of PHM strategies for cables.

Alternative solutions based on operational datasets have been developed such as cable prognostics modelling based on voltage and current [8], internet-of-things based partial discharge sensing networks [9], cable condition monitoring through power line modems, time-frequency reflectometry and machine learning strategies [10], and subsea power cable degradation analysis due to corrosion and abrasion [11].

The interest of some other data-driven methods for cable failure specification has been on designing statistical distributions to model the cable lifetime through a parametric function. These models can be used to estimate cable failure occurrences [12] and plan system-level cable maintenance strategies integrated within power system models [13], [14].

2) Physics-of-failure PHM solutions: Due to the limited availability of run-to-failure and diagnostics data, physics-offailure $(\mathrm{PoF})$ models have attracted a wide interest [15], [16], [17]. There are different factors that influence the remaining useful life (RUL) of a cable, and accordingly, the specification of an accurate physics-based model for RUL prediction is a challenging task. In this context, the focus of many PoF models has been on calculating the cable conductor temperature through different techniques such as finite element methods [18] or equivalent electro-thermal circuits [15], [16], [17]. With the estimated cable temperature it is possible to calculate the associated lifetime reduction due to thermal stress through lifetime models [17], [19], [20].

The cable temperature estimation is a widely addressed research area with applications in dynamic line rating [21], [22], [23] and lifetime estimation [24]. In addition to the thermal stress, there are models focused on the analysis of the joint influence of electrical and thermal stresses [17]. Many of these models have been validated via experimental field tests [25].

3) Uncertainty analysis: The presence of uncertainties in cable temperature and lifetime estimation has been discussed by different authors [2]. The increased availability of data and 
the emergence of data-driven solutions has contributed to the proliferation of uncertainties due to the use of diverse data collection equipment with associated errors, e.g. calibration or conversion errors (measurement uncertainty) and health estimation algorithms which adopt modelling assumptions (process uncertainty).

The cable lifetime modelling process encompasses different sources of uncertainty and there are different methods that can be used to integrate uncertainty criteria in the cable temperature and lifetime modelling processes [26]. Generally, uncertainty modelling methods can be classified into interval theory, fuzzy set based methods and probabilistic approaches.

Villacci and Vaccaro integrated measurement uncertainties in the cable thermal model through interval mathematics [27]. It provides fast processing times when probabilistic information is not available. Wang and Qiu used fuzzy logic to integrate uncertainty information in the cable thermal model [28], which requires the formalization of expert knowledge via membership functions. The focus of this work is on data-driven probabilistic models that do not require the formalization of expert knowledge. Shabani and Vahidi presented an optimization approach so as to maximize ampacity and minimize installation costs [29]. The method solves a deterministic objective function and takes into account the uncertainty of ambient temperature, load and soil via Monte Carlo simulations (MCS). However, MCS lacks a systematic framework to integrate and propagate uncertainties not only for variables, but also for modelling processes.

\section{B. Research direction and contribution}

The lifetime reduction of a power cable varies non-linearly according to different stochastic variables, state-estimation and degradation models, and the sources of uncertainty associated with these variables and evaluation models.

If these factors are not integrated in an adequate cable health state estimation framework this may limit the application of PHM strategies for effective cable lifetime estimation under uncertainty and it may lead to non-optimal cable maintenance decisions. Due to the complex PoF degradation process of cables, deterministic models or simplified PoF models are generally adopted resulting in non-accurate decision-making under uncertainty [30].

In contrast, the integration of uncertainties leads to a probabilistic decision-making process. This change of paradigm impacts directly on the flexibility to adopt decisions. An uncertainty-aware framework propagates the variability of modelling and measurement parameters to the final health state and infers confidence intervals to aid decision-making under uncertainty. The probabilistic confidence bounds associated with the lifetime estimation assist the engineer in the final decision-making process with information about the confidence of the estimate. That is, if confidence bounds are wide and the probability of the maximum likelihood value is low, it reflects a complex decision-making situation where the model is not confident about the final estimate. In contrast, narrow confidence bounds with a high probability indicate a high-confidence scenario for decision-making.
From a review of the literature, it appears that an effective uncertainty processing framework for cable lifetime estimation has not been proposed, which integrates systematically different sources of uncertainty and propagates them to the final cable health state estimation for decision-making under uncertainty.

To cover this gap, the contribution of this paper is the implementation of a cable diagnostics framework that connects data-driven probabilistic uncertainty models with PoFbased operation and degradation models for the cable lifetime evaluation under uncertainty. The framework integrates and propagates measurement and process modelling errors in the cable conductor temperature and lifetime estimation models and infers the cable health state under different operation and error scenarios. In addition, the sensitivity evaluation of the measurement errors is also developed to improve the decisionmaking process.

Accordingly, a cable diagnostics framework is presented based on Particle Filtering (PF) concepts [31] integrating nonlinear state-space lifetime equations with physics-based equations. The state-space model dynamically updates the power cable lifetime using loading and temperature profiles along with physical configuration parameters through a physicsbased model. This model estimates the conductor temperature according to design and construction parameters. The cable lifetime is then estimated as a probability density function (pdf) obtained from the posterior distribution elicited from the PF method. Subsequently $95 \%$ confidence intervals are elicited from the obtained pdfs to assist the decision maker in the visualization and interpretation of the results.

\section{Organization}

The rest of this paper is organised as follows. Section II introduces cable temperature and lifetime estimation methods. Section III defines the proposed approach for cable health state estimation under uncertainty. Section IV presents evaluations and results. Section $\mathrm{V}$ discusses the challenges and potential extensions, and finally Section VI draws conclusions.

\section{CABle Thermal \& Lifetime Modelling}

The cable conductor temperature is directly influenced by its layers and materials, physical configuration and surrounding environment. In turn, the temperature impacts on the cable lifetime along with other parameters such as electrical stress.

\section{A. Thermal modelling}

The cable temperature can be calculated from analytic methods [15], [16], [17], finite element methods (FEM) [18] or non-invasive inspection measurements [32]. The expensive temperature measuring equipment is not cost-effective and the implementation of FEM is outside the scope of this work. Therefore this work focuses on analytic methods [2], [33].

Fig. 1a shows the equivalent thermal ladder circuit of a cable from the inner conductor layer to the external jacket.

The temperature $\Theta$ of each layer $l$ of the cable, $\Theta_{l}$, is modelled through the thermal resistance $\left(T_{l}\right)$, thermal capacitance $\left(Q_{l}\right)$, and power loss unit $\left(W_{l}\right)$. 


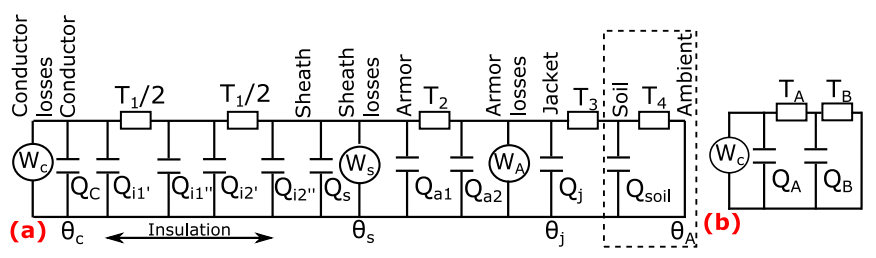

Fig. 1. (a) Thermal ladder circuit of a cable. (b) Equivalent two-loop circuit.

1) Thermal resistance \& capacitance: The thermal resistance models the heat dissipated in the cable:

$$
T=\frac{\rho}{2 \pi} \log \left(D_{\text {ext }} / D_{\text {int }}\right)
$$

where $\rho$ is the thermal resistivity of the cable layer's material (K.m/W), and $D_{\text {ext }}$ and $D_{\text {int }}$ are the external and internal diameters of the corresponding cable layer under study. For example, the thermal resistance of the insulator, $T_{\text {ins }}$, is typically calculated by taking the diameters of the cable conductor and sheath: $T_{\text {ins }}=\frac{\rho}{2 \pi} \log \left(D_{\text {sheath }} / D_{\text {cond }}\right)$

The thermal capacitance models the capacity of the cable to hold heat and it is calculated as follows:

$$
Q=\pi / 4\left(D_{\mathrm{ext}}{ }^{2}-D_{\mathrm{int}}{ }^{2}\right) C
$$

where $C$ is the volumetric specific heat of the cable layer's material $\left(\mathrm{J} / \mathrm{m}^{3 \circ} \mathrm{C}\right)$ and $D_{\text {ext }}$ and $D_{\text {int }}$ are defined immediately above. For example, the thermal capacitance of the sheath, $Q_{\text {sheath }}$, is calculated by taking the diameters of the insulator and sheath: $Q_{\text {sheath }}=\pi / 4\left(D_{\text {sheath }}{ }^{2}-D_{\text {ins }}^{2}\right) C$.

2) AC Resistance: The AC resistance models the resistance of the cable material to the heat. The alternating current resistance of the cable, $R_{\mathrm{AC}}$, is calculated as follows:

$$
R_{\mathrm{AC}}=R_{\mathrm{DC}}\left(1+y_{s}+y_{p}\right)
$$

where $R_{\mathrm{DC}}$ is the direct current resistance of the cable $(\Omega / \mathrm{m})$, and $y_{s}$ and $y_{p}$ are the skin and proximity effect factors respectively. The direct current resistance, $R_{\mathrm{DC}}$, is defined as:

$$
R_{\mathrm{DC}}=\rho_{20} / S\left(1+\alpha_{\text {mat. }}(90-20)\right)
$$

where $\rho_{20}$ is the resistivity of the material at $20{ }^{\circ} \mathrm{C}(\Omega . \mathrm{m})$, $\alpha_{\text {mat. }}$ is the temperature coefficient of the material $\left(K^{-1}\right)$, and $S$ is the cross-section area of the conductor $\left(\mathrm{m}^{2}\right)$.

The skin effect, $y_{s}$, is the tendency of an AC current to become distributed such that the current density is largest near the surface of the conductor and decreases with greater depths in the conductor. It is defined as:

where,

$$
y_{s}=x_{s}^{2} /\left(192+0.8 x_{s}^{2}\right)
$$

$$
x_{s}=F_{k} k_{s} ; \quad F_{k}=8 \pi f e^{-7} / R_{\mathrm{DC}}
$$

where $f$ is the operating frequency.

The proximity effect can increase the AC resistance of adjacent conductors by inducing eddy currents. Proximity effect depends on the cable configuration. Assuming cables in trefoil formation, the proximity factor, $y_{p}$, is obtained from:

$$
y_{p}=F_{p}\left((0.312+1.18) /\left(F_{p}+0.27\right)\right)
$$

where,

$$
F_{p}=x_{p}^{2} /\left(192+0.8 x_{p}^{2}\right) ; \quad x_{p}=F_{k} k_{p}
$$

where $k_{p}$ and $k_{s}$ are directly determined from experimental tables depending on cable configuration and material [2].
3) Losses: The cable power loss is directly translated into dissipated heat and increased cable temperature. The total cable temperature is the sum of self-heating, mutual heating between adjacent cables and the temperature rise due to the soil effect. The total cable temperature is then translated into cable power loss, which is generally modelled according to the different layers of the cable (cf. Fig. 1a).

Assuming a cable comprised of conductor, jacket and armour, the power loss due to self-heating is calculated as:

$$
W_{\text {self }}(t)=W_{c}(t)+W_{s}(t)+W_{j}(t)+W_{a}(t)
$$

where $W_{c}$ is the conductor power loss, and $W_{s}, W_{j}$ and $W_{a}$ are respectively the sheath, jacket and armour power losses.

4) Conductor losses: The conductor power loss at time $t$, $W_{c}(t)$, is defined as follows:

$$
W_{c}(t)=I(t)^{2} R_{\mathrm{AC}}
$$

where $I(t)$ is the current that flows through the cable at time instant $t$ and $R_{\mathrm{AC}}$ is the alternating resistance of the cable.

5) Sheath and jacket/armour losses: Sheath losses depend on the cable configuration. For cables in trefoil formation with metallic sheath, loss factors in the sheath and jacket/armour are defined as:

$$
\lambda=\lambda_{1}+\lambda_{2}
$$

where $\lambda$ are the total losses in the sheath and jacket, $\lambda_{1}$ is the relation of the total losses in the sheath with respect to the total losses in all the cable conductors, $\lambda_{2}$ is the relation of the total losses in the jacket/armour with respect to the total losses in all the cable conductors.

Depending on the cable configuration the calculation of $\lambda_{1}$ is different. For three single-core cables in trefoil formation:

$$
\lambda_{1}=R_{s} / R_{\mathrm{AC}}\left(1 /\left(1+R s / X^{2}\right)\right)
$$

where $R_{s}$ is the sheath's resistance and $X$ is the sheath's reactance per unit length $(\Omega / \mathrm{m})$ defined as:

$$
X=2 \omega e^{-7} \cdot \log \left(2 D_{\text {axis }} / D_{s}\right)
$$

where $D_{\text {axis }}$ is the distance between conductor axes of the cables $(\mathrm{mm})$, and $D_{s}$ is the mean diameter of the sheath $(\mathrm{mm})$.

Accordingly, the sheath and jacket/armour power loss are calculated as follows:

$$
W_{s}=\lambda_{1} W_{c}, \quad W_{j}=\lambda_{2} W_{c}
$$

Finally (9) can be redefined as $W_{\text {self }}(t)=W_{c}(t)\left(1+\lambda_{1}+\lambda_{2}\right)$. In this analysis it is assumed that the cable has no jacket and therefore $\lambda_{2}=0$ and $W_{\text {self }}(t)=W_{c}(t)\left(1+\lambda_{1}\right)$.

6) Dielectric loss: For AC cables the dielectric loss is defined as follows:

$$
W_{d}=\omega C U_{0}^{2} \tan \delta
$$

where $\omega=2 \pi f, C$ is the capacitance per unit length $(\mathrm{F} / \mathrm{m})$, and $U_{0}$ is the voltage to earth. For distribution voltages the effect of dielectric losses can be neglected [20] and accordingly it has not been included in this paper. 
7) Transient Operation: The transient response of the cable is calculated using the Van Wormer coefficients [2]:

$$
p^{*}=\frac{1}{\log \left(\frac{D_{\text {ins }}}{D_{\text {cond }}}\right)}-\frac{1}{\frac{D_{\text {ins }}}{D_{\text {cond }}}} ; p^{\prime}=\frac{1}{2 \log \left(\frac{D_{\text {ext }}}{D_{\text {sheath }}}\right)}-\frac{1}{\left(\frac{D_{\text {ext }}}{D_{\text {sheath }}}\right)^{2}-1}
$$

Short duration insulation capacitances are defined as [2]:

$$
\begin{aligned}
& Q_{I 1}=\pi / 4\left(D_{\text {ins. }} D_{\text {cond. }}-D_{\text {cond. }}{ }^{2}\right) c_{\text {ins. }} . \\
& Q_{I 2}=\pi / 4\left(D_{\text {ins. }}{ }^{2}-D_{\text {ins. }} D_{\text {cond. }}\right) c_{\text {ins. }}
\end{aligned}
$$

When modelling transient operation it is suggested to divide the insulation in 2 additional sublayers with thermal resistivity $T_{1} / 2$ (see insulation in Fig.1a). Accordingly, based on (16)(17) the short-term insulator response is defined as [2]:

$$
\begin{array}{ll}
Q_{i 1^{\prime}}=p^{*} Q_{I 1} ; & Q_{i 1^{\prime \prime}}=\left(1-p^{*}\right) Q_{I 1} \\
Q_{i 2^{\prime}}=p^{*} Q_{I 2} ; & Q_{i 2^{\prime \prime}}=\left(1-p^{*}\right) Q_{I 2}
\end{array}
$$

Applying the same division to the shield, armour and jacket:

$$
\begin{aligned}
& Q_{a 1}=p^{*} Q_{\text {armor }} ; \quad Q_{a 2}=\left(1-p^{*}\right) Q_{\text {armor }} \\
& Q_{j 1}=p^{\prime} Q_{\text {jacket }} ; \quad Q_{j 2}=\left(1-p^{\prime}\right) Q_{\text {jacket }}
\end{aligned}
$$

where $Q_{\text {jacket }}, Q_{\text {armor }}, Q_{\text {shield }}$ are calculated through (2).

In order to reduce the ladder circuit in Fig.1 a to a two-loop equivalent circuit shown in Fig.1b, capacitances are joined per each section of the equivalent ladder circuit, i.e. sheath's capacitance $q_{s}=1+\lambda_{1}$ and jacket's capacitance $q_{j}=1+\lambda_{1}+\lambda_{2}$. Namely the capacitances are grouped as follows:

$$
\begin{array}{ll}
Q_{1}=Q_{c}+Q_{i 1} ; & Q_{2}=Q_{i 2}+Q_{i 3} \\
Q_{3}=Q_{i 4}+Q_{\text {sheath }} / q s+Q_{a 1} / q s ; & Q_{4}=\left(Q_{a 2}+Q_{j 1}\right) / q s
\end{array}
$$

Accordingly, the ladder circuit in Fig. 1a is reduced to its equivalent circuit shown in Fig. $1 \mathrm{~b}$ to resolve the temporal response. These are the parameters of the reduced circuit [2]:

$$
\begin{array}{ll}
T_{A}=T_{1} / 2 ; & T_{B}=T_{1} / 2+q_{s} T_{2}+q_{j} T_{3} \\
Q_{A}=Q_{1} ; & Q_{B}=Q_{2}+Q_{3}\left(\left(q_{s} T_{2}+q_{j} T_{3}\right) / T_{B}\right)^{2}+Q_{4}\left(q_{j} T_{3} / T_{B}\right)^{2}
\end{array}
$$

8) Temporal Response: The circuit in Fig. $1 \mathrm{~b}$ is solved using the Laplace transform as follows:

$$
H(s)=\frac{T_{A}+T_{B}+T_{A} T_{B} Q_{B} s}{1+s\left(T_{A} Q_{A}+T_{B} Q_{B}+T_{B} Q_{A}\right)+s^{2}\left(T_{A} Q_{A} T_{B} Q_{B}\right)}
$$

where $s$ is a complex number frequency parameter $s=\sigma+$ $i \omega$, and $F(s)=\int_{0}^{\infty} f(t) e^{-s t} d t$. The response of the transfer function in (22) models the temporal response of the cable conductor temperature due to the self-heating of the cable.

Changing from $s$ to the temporal domain, $t$, the temporal response of (22), i.e. cable conductor temperature due to selfheating, $\Theta_{\text {self }}(t)$, is defined as follows:

$$
\Theta_{\text {self }}(t)=W_{c}\left(T_{a}\left(1-e^{-a t}\right)+T_{b}\left(1-e^{-b t}\right)\right)
$$

where the temporal response parameters are defined as follows:

$$
\begin{aligned}
& T_{a}=(1 /(a-b))\left(1 / Q_{A}-b\left(T_{A}+T_{B}\right)\right) ; \quad T_{b}=T_{A}+T_{B}-T_{a} \\
& a=\left(M_{0}+\sqrt{M_{0}^{2}-N_{0}}\right) / N_{0} ; \quad b=\left(M_{0}-\sqrt{M_{0}^{2}-N_{0}}\right) / N_{0}
\end{aligned}
$$

where $T_{A}, T_{B}, Q_{A}$, and $Q_{B}$ are defined in (21), and:

$$
\begin{aligned}
& M_{0}=1 / 2\left(Q_{A}\left(T_{A}+T_{B}\right)+Q_{B} T_{B}\right) \\
& N_{0}=Q_{A} T_{A} Q_{B} T_{B}
\end{aligned}
$$

The soil temperature is crucial for underground power cables because it directly affects the final cable temperature. The soil temperature rise, $\Theta_{\text {soil }}(t)$, due to constant power dissipation transient, $W_{\text {self }}$, is defined as:

$$
\Theta_{\text {soil }}(t)=\alpha(t)\left[W_{\text {self }} \frac{\rho_{s}}{4 \pi}\left(E_{i}\left(\frac{-L^{2}}{\sigma t}\right)-E_{i}\left(\frac{-D_{\text {surf }}^{2}}{16 \sigma t}\right)\right)\right]
$$

where $L$ denotes the depth burial of the cable $(\mathrm{m}), D_{\text {surf }}$ denotes the external surface diameter of the cable $(\mathrm{m}), \sigma$ denotes the diffusivity of the soil $\left(\mathrm{m}^{2} / \mathrm{s}\right), W_{\text {self }}$ can be calculated from $(9)$, and $E_{i}($.$) is the exponential integral function.$

With more than one cable located next to each other, the proximity of the cables causes mutual heating. The mutual heating temperature, $\Theta_{\text {mutual }}(t)$, is defined as follows:

$$
\Theta_{\text {mutual }}(t)=\alpha(t) \sum_{k=1}^{N-1}\left[W_{\text {self }} \frac{\rho_{s}}{4 \pi}\left(E_{i}\left(\frac{-d_{\mathrm{pk}^{2}}^{2}}{4 \sigma t}\right)-E_{i}\left(\frac{-d_{\mathrm{pk}^{\prime}}}{4 \sigma t}\right)\right)\right]
$$

where $d_{\mathrm{pk}}$ denotes the distance from the cable $p$ to cable $k$ (m) and $d_{\mathrm{pk}}$ is the virtual distance from the cable $p$ to $k(\mathrm{~m})$.

The attainment factor, $\alpha(t)$, is defined as follows:

$$
\alpha(t)=\frac{\Theta_{\text {self }}(t)}{W_{c}(t)\left(T_{A}+T_{B}\right)}=\frac{T_{a}\left(1-e^{-a t}\right)+T_{b}\left(1-e^{-b t}\right)}{T_{A}+T_{B}}
$$

The total rise of temperature over ambient temperature is defined as follows:

$$
\begin{aligned}
& \Theta_{\text {rise }}(t)=\Theta_{\text {self }}(t)+\Theta_{\text {soil }}(t)+\Theta_{\text {mutual }}(t)= \\
& W_{\mathrm{c}}(t)\left(T_{a}\left(1-e^{-a t}\right)+T_{b}\left(1-e^{-b t}\right)\right)\left[1+\frac{\rho_{s}}{4 \pi} \frac{\left(1+\lambda_{1}\right)}{\left(T_{A}+T_{B}\right)}\right. \\
& \left.\left(\left[E_{i}\left(\frac{-L^{2}}{\sigma t}\right)-E_{i}\left(\frac{-D_{\text {surf }}^{2}}{16 \sigma t}\right)\right]+2\left[E_{i}\left(\frac{-d_{p k}^{2}}{4 \sigma t}\right)-E_{i}\left(\frac{-d_{p k^{\prime}}^{2}}{4 \sigma t}\right)\right]\right)\right]
\end{aligned}
$$

In order to account for the temperature changing alternating current resistance, a correction factor is applied to the final temperature rise over ambient, $\Theta_{\text {rise, },}(t)$ :

$$
\Theta_{\text {rise, } C}(t)=\Theta_{\text {rise }}(t) /\left(1+\frac{\Theta(\infty)-\Theta_{\text {Rise }}(t)}{234.5+\Theta_{\text {amb. }}(t)}\right)
$$

The total stationary temperature, $\Theta(\infty)$, is calculated as:

$$
\begin{aligned}
\Theta(\infty) & =\Theta_{\text {self }}(\infty)+\Theta_{\text {mutual }}(\infty)+\Theta_{\text {soil }}(\infty) \\
\Theta_{\text {self }}(\infty) & =W_{c}(t)\left(T_{A}+T_{B}\right) \\
\Theta_{\text {mutual }}(\infty) & =W_{\text {self }}(t) \rho_{s} / 2 \pi \log \left(k p^{\prime} / k p\right) \\
\Theta_{\text {soil }}(\infty) & =W_{\text {self }}(t) \rho_{s} / 2 \pi \log \left(2 L / D_{\mathrm{s}}+\sqrt{4 L^{2} / D_{\mathrm{s}}^{2}}-1\right)
\end{aligned}
$$

where $L$ is the depth burial of the cable, and $D_{\mathrm{s}}$ is the surface. After reorganising and reducing the total stationary temperature can be redefined as:

$$
\Theta(\infty)=W_{c}(t)\left\{\left(T_{A}+T_{B}\right)+\frac{\left(1+\lambda_{1}\right) \rho_{s}}{2 \pi}\left[\log \left(\frac{k p^{\prime}}{k p}\right)+\log \left(\frac{2 L}{D_{\mathrm{s}}} \sqrt{d}\right)\right]\right\}
$$

where $d=4 L / D_{s}^{2}-1$.

Finally, the cable temperature is determined from:

$$
\Theta_{\text {cable }}(t)=\Theta_{\text {amb. }}(t)+\Theta_{\text {rise, }}(t)
$$

Algorithm 1 defines the cable temperature estimation framework for the time interval $\left[k_{0}, K\right]$ : 
- Lines 1-5: define the temperature-independent cable parameters which depend on the physical characteristics.

- Lines 6-12: estimate the cable temperature for the diagnostics time horizon $K$. It is assumed that the load, $i$, and the ambient temperature, $\Theta_{a m b}$, measurements are collected every $\Delta \mathrm{t}$ within the time interval $\left[k_{0}, K\right]$ and superposition of past transients are applied.

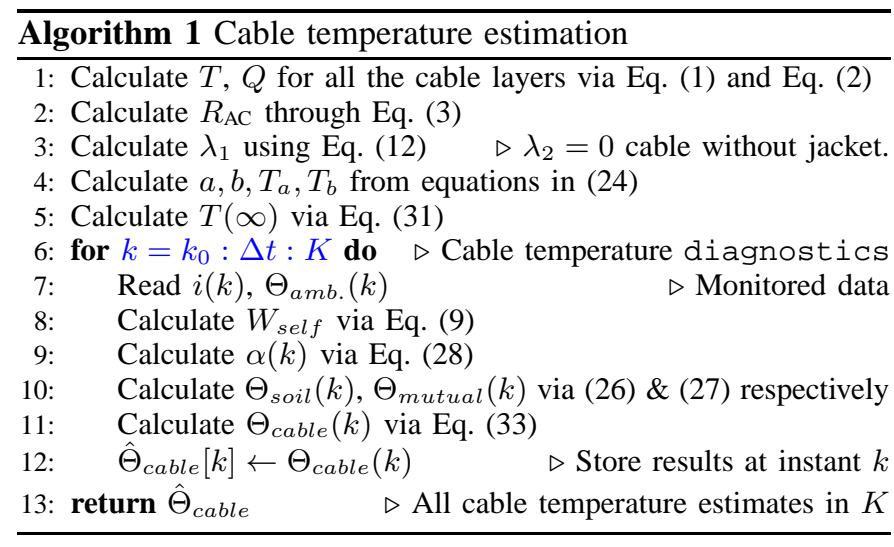

\section{B. Lifetime estimation}

There are different lifetime models that diagnose the cable health based on thermal and electrical stresses [19]. This paper focuses on the Arrhenius inverse power model (IPM) due to its wide adoption in the community, e.g [34], [35]. IPM defines the cable time-to-failure (TTF) as follows [19]:

$$
T T F=\mathrm{TTF}_{0} e^{-B c T} E / E_{0}^{-\left(\eta_{0}-b c T\right)}
$$

where $E$ is the electric field $(\mathrm{kV} / \mathrm{mm}), c T=1 / T_{0}-1 / T$ is the conventional thermal stress, where $T$ is temperature in Kelvin degrees and $T_{0}$ is a reference temperature, $\eta_{0}$ is the voltage endurance coefficient at $T=T_{0}$ (non-dimensional), $E_{0}$ is a value of electric field below which electrical aging is deemed as negligible $(\mathrm{kV} / \mathrm{mm}), \mathrm{TTF}_{0}$ is time-to-failure at $T=T_{0}$ and $E=E_{0}, B$ is equal to $\Delta W / k$ ( $\Delta W$ being the activation energy of the main thermal degradation reaction and $k$ being the Boltzman constant) and $b$ is a parameter that models the synergism between electrical and thermal stresses (K.mm/kV).

In order to estimate the remaining useful life (RUL) iteratively (34) has been rewritten as a recurrence relation form:

$$
R U L_{t}=R U L_{t-1}-\Delta t / T T F
$$

where $R U L_{t}$ is the cable RUL at $t, R U L_{t-1}$ is the RUL at $t-1, \Delta \mathrm{t}$ is the time-step, and $T T F$ is the time-to-failure defined in (34). The fraction $\Delta t / T T F$ models the loss-of-life fraction in each iteration. At instant $t=0, R U L_{t-1}$ equals to the initial lifetime estimation $R U L_{0}$. In subsequent iterations $R U L_{0}$ is updated with the most up-to-date RUL estimation to reflect the previous state at $t-1$.

\section{Sources of uncertainty}

Algorithm 1 is the method used by the IEC standard and generally adopted by the research conducted around cable conductor temperature estimation [15], [16], [17]. However, some measurements may be affected by hardware inaccuracies or calibration errors, modelling imprecision errors may also influence the final estimation, and in the end these factors will impact on the accuracy of the final estimate. Under conditions of errors and uncertainty, the estimation of a single point cable conductor temperature may not be accurate. A pdf around a number of conductor temperature values with the probability associated with each value represents better the uncertain nature of the cable conductor temperature estimate. Some examples include measurement errors due to sensor calibration or noisy environments, lack of exact knowledge of empirical formulations, and physical configuration uncertainties such as the exact distance to the cable or the exact soil diffusivity.

In this context, given the uncertainties around the cable temperature estimate, it is possible to re-evaluate the lifetime models to quantify effect of cable temperature estimation errors on cable lifetime estimation.

The lifetime equation can be redefined as:

$$
R U L_{t}=R U L_{t-1}+\nu_{R U L_{t-1}}-\Delta t /\left(T T F+\nu_{t}\right)
$$

where $\nu_{R U L_{t-1}}$ is the RUL estimate error at $t-1$ and $\nu_{t}$ is the process error. At the initial time instant $\nu_{R U L_{t-1}}$ will denote the uncertainty around the initial life estimate $\nu_{R U L_{0}}$ and this will be propagated in subsequent iterations.

The uncertainty around the loss-of-life fraction $\Delta t / T T F$ is defined in terms of the process and measurement uncertainties. The main measurement sources of uncertainty are load, $\psi_{I}$, and ambient temperature, $\psi_{a}$, measurements. Integrating uncertainty measurements in (33) results in:

$$
\Theta_{\text {cable }}(t)=\Theta_{a m b}(t)+\psi_{a}+\frac{\Theta_{r i s e}(t)\left(234.5+\Theta_{a m b}(t)+\psi_{a}\right)}{1+\frac{\Theta(\infty)-\Theta_{\text {rise }}(t)}{234.5+\Theta_{a m b}(t)+\psi_{a}}}
$$

defining $f_{1}(t)$ and $f_{2}(t)$ as follows:

$$
\begin{aligned}
& f_{1}(t)=\left[T_{a}\left(1-e^{-a t}\right)+T_{b}\left(1-e^{-b t}\right)\right] \\
& {\left[1+\frac{\left(1+\lambda_{1}\right) \rho_{s}}{4 \pi}\left(\left[E_{i}\left(\frac{-L^{2}}{\sigma t}\right)-E_{i}\left(\frac{D_{\text {surf }^{2}}}{16 \sigma t}\right)\right]+2\left[E_{i}\left(\frac{-d_{p k}^{2}}{4 \sigma t}\right)-E_{i}\left(\frac{-d_{p k^{\prime}}^{2}}{4 \sigma t}\right)\right]\right.\right.} \\
& f_{2}(t)=\left(T_{A}+T_{B}\right)+\frac{\left(1+\lambda_{1}\right) \rho_{s}}{2 \pi}\left(\log \left(\frac{d_{k p^{\prime}}}{d_{k p}}\right)+\log \left(\frac{2 L}{D_{\text {surf }}} \sqrt{\left.\left.\frac{4 L}{D_{\text {surf }}^{2}}-1\right)\right)}\right.\right.
\end{aligned}
$$

results in:

$$
\Theta_{\text {cable }}(t)=\Theta_{a m b}(t)+\psi_{a}+\frac{f_{1}(t)\left[234.5+\Theta_{a m b}(t)+\psi_{a}\right]^{2}}{\frac{234.5+\Theta_{a m b}(t)+\psi_{a}}{R_{\mathrm{AC}}\left(I(t)+\psi_{I}\right)^{2}}+f_{2}(t)-f_{1}(t)}
$$

Comparing (40) with (33) it is possible to see that the different uncertainty sources may affect the cable temperature and accordingly, comparing (36) with (35) it is possible to see that the lifetime estimation is also affected by different sources of uncertainty. The proposed framework below effectively integrates these sources of uncertainty.

\section{A Novel Diagnostics Framework for CABle LIFETIME ESTIMATION UNDER UNCERTAINTY}

Power cable aging can be considered a partially-observable Markovian process, where the state of the cable cannot be directly observed, but it can be estimated from measurands like ambient temperature and load. Accordingly, so as to take into account different uncertainties and propagate them along with inspection data, the PF method has been implemented. 
The PF is a Monte Carlo based Bayesian filtering method [31], which has been used for prognostics predictions of transformers using machine learning strategies with different measurement and degradation functions and sources of uncertainty [36]. PF enables the estimation of cable lifetime under different sources of uncertainty as shown in Fig. 2.

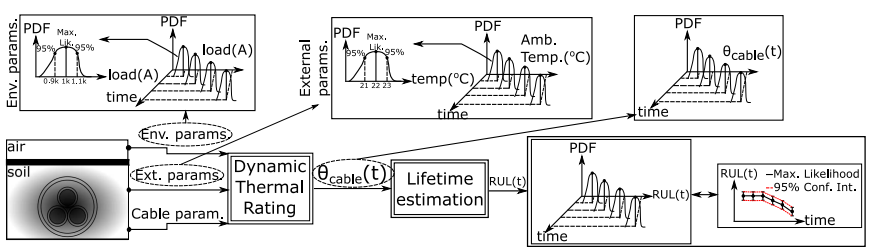

Fig. 2. High-level PF-based cable lifetime diagnostics framework.

The Dynamic Thermal Rating model in Fig. 2 is Algorithm 1 adapted for uncertainty measurements according to the PF approach. It takes as input environmental parameters, cable parameters and external parameters which can be specified with pdfs to model the associated uncertainty, e.g. ambient temperature and load. The outcomes of this model are a number of pdfs (one per each evaluated data point) of the cable temperature, $\Theta_{\text {cable. }}$ The Lifetime Estimation model evaluates the cable RUL, $R U L(t)$, with input cable conductor temperature estimate pdfs and calculates the lifetime pdfs (one per each evaluated data point). Finally the pdfs are converted into maximum likelihood and $95 \%$ confidence intervals so as to improve the readability for the end-user.

\section{A. Particle Filtering for cable diagnostics}

The parametric state-space formulation for a dynamic model at the discrete-time instant $k$ is described as follows [31]:

$$
\begin{aligned}
& x_{\mathrm{k}}=f\left(x_{\mathrm{k}-1}, \nu_{\mathrm{k}}\right) \quad \text { "Degradation equation" } \\
& z_{\mathrm{k}}=h\left(x_{\mathrm{k}}, \psi_{\mathrm{k}}\right) \quad \text { "Measurement equation" }
\end{aligned}
$$

where $x_{k}$ is the unobserved cable health state vector, $z_{k}$ is the measurements vector, $f(\cdot)$ is the degradation function, $h(\cdot)$ is the measurement function, $\nu_{\mathrm{k}}$ is the process noise vector, and $\psi_{\mathrm{k}}$ is the measurement noise vector. Each element of the noise vectors represent a different source of uncertainty, and each of them is formalized through its corresponding pdf $\nu_{\mathrm{k}} \sim p_{\nu}$ and $\psi_{\mathrm{k}} \sim p_{\psi}$. The uncertainty sources considered in this work are synthesized as follows $\nu_{\mathrm{k}}=\left\langle\nu_{\mathrm{k}}, \nu_{\mathrm{RUL}_{0}}\right\rangle$ and $\psi_{\mathrm{k}}=\left\langle\psi_{\mathrm{a}}, \psi_{\mathrm{I}}\right\rangle$.

The resolution of this state-space problem enables filtering the cable health state at the instant $k$ integrating multiple sources of uncertainty [31]. Fig. 3 shows the application of (41) to the cable lifetime estimation process.

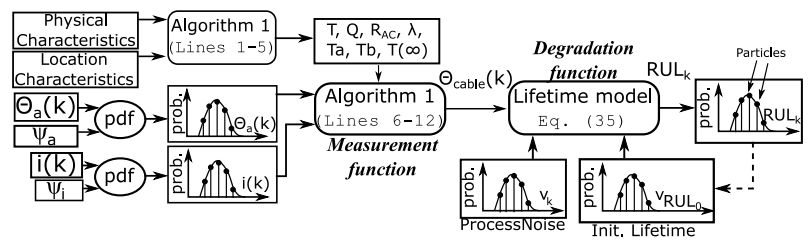

Fig. 3. Framework for cable lifetime diagnostics under uncertainty.

The measurement equation is defined by the cable conductor temperature calculated according to Algorithm 1. The measurements included in the cable conductor temperature are load, $i(k)$, and ambient temperature, $\Theta_{\mathrm{amb}}(k)$, along with their measurement errors, $\psi_{\mathrm{I}}$ and $\psi_{\mathrm{a}}$, respectively, and other cable parameters and physical characteristics, which result in the calculation of the cable conductor temperature $\Theta_{\text {cable }}(k)$.

The cable health state at the discrete instant $k, R U L_{k}$, is calculated via (36). This equation defines the cable degradation trend as a function of the cable temperature, electrical stress, the synergism between thermal and electrical stresses, and cable health state at $k-1$, which is updated at every time-step with the most up-to-date cable health state estimation.

The pdf $p\left(x_{\mathrm{k}} \mid z_{0: \mathrm{k}}\right)$ defines the system state $x_{\mathrm{k}}$ with measurements until the instant $k, z_{\mathrm{k}}$. The prior pdf of $x_{\mathrm{k}}$ from $p\left(x_{\mathrm{k}-1} \mid z_{0: \mathrm{k}-1}\right)$ is determined as follows [31]:

$$
p\left(x_{\mathrm{k}} \mid z_{0: \mathrm{k}-1}\right)=\int p\left(x_{\mathrm{k}} \mid x_{\mathrm{k}-1}\right) p\left(x_{\mathrm{k}-1} \mid z_{0: \mathrm{k}-1}\right) d x_{\mathrm{k}-1}
$$

where (41) defines the cable state-transition $p\left(x_{\mathrm{k}} \mid x_{\mathrm{k}-1}\right)$. The prior pdf is updated with new measurements gathered at instant $k, z_{0: k}$, leading to the posterior pdf [31]:

$$
p\left(x_{\mathrm{k}} \mid z_{0: \mathrm{k}}\right)=p\left(x_{\mathrm{k}} \mid z_{0: \mathrm{k}-1}\right) p\left(z_{\mathrm{k}} \mid x_{\mathrm{k}}\right) / p\left(z_{\mathrm{k}} \mid z_{0: \mathrm{k}-1}\right)
$$

For each instant $k$, the PF method applies prediction, update and resampling steps so as to solve (43). Particles are a key concept for the resolution, which are random samples with their weights that describe a pdf, e.g. $\left\{x_{k-1}^{i}, w_{k-1}^{i}\right\}_{i=1}^{N_{\mathrm{p}}}$ denotes the system state at time $k-1$ with $N_{\mathrm{p}}$ particles.

Algorithm 2 defines the implementation of the cable RUL prediction under uncertainty.

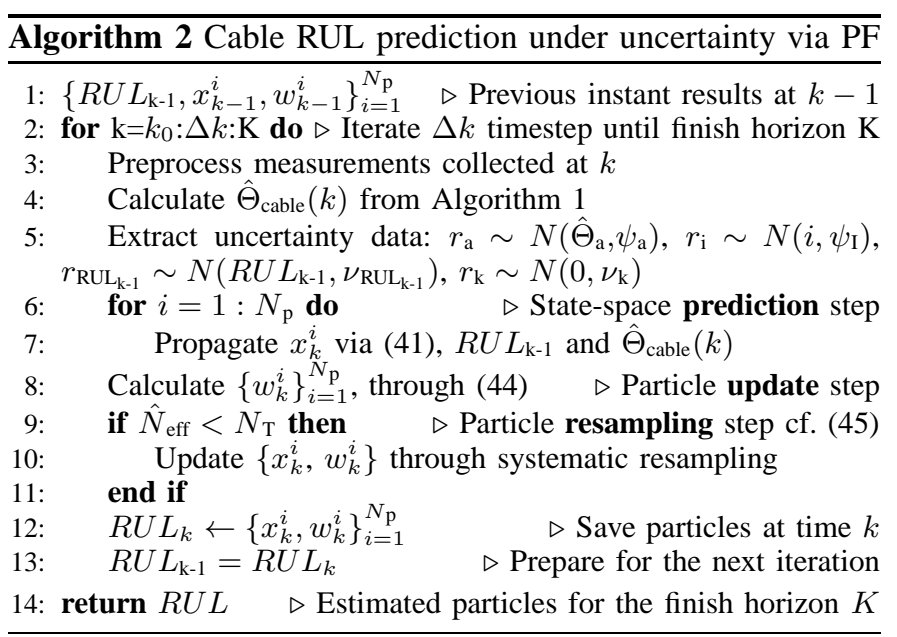

The prediction at $k$ is done by simulating the cable dynamics according to (41) to generate new samples $x_{k}^{i}$ from the predicted distribution $p\left(x_{\mathrm{k}} \mid z_{0: \mathrm{k}-1}\right)$ ( 1 ines $6-7$ ).

Next, measurement likelihoods are calculated given the health state of the $\mathrm{i}$-th particle $p\left(z_{k} \mid x_{k}^{i}\right)$, and accordingly, a weight is estimated for every particle $\mathrm{i}=\left\{1, \ldots, N_{p}\right\}$ (line 8):

$$
w_{k}^{i}=p\left(z_{\mathrm{k}} \mid x_{k}^{i}\right) / \sum_{j=1}^{N_{\mathrm{p}}} p\left(z_{\mathrm{k}} \mid x_{k}^{j}\right)
$$

The obtained set of particles $\left\{x_{k}^{i}, w_{k}^{i}\right\}_{i=1}^{N_{p}}$ are then used to elicit the posterior pdf $p\left(x_{\mathrm{k}} \mid z_{0: \mathrm{k}}\right)$.

The PF introduces the weight degeneracy issue [31]: one particle has meaningful weight and the rest are negligible. An effective particle size is defined to eliminate the degeneracy:

$$
\hat{N}_{\text {eff }}=1 / \sum_{i=1}^{N_{\mathrm{p}}} w_{k}^{i}
$$


The systematic-resampling is applied if $\hat{N}_{\text {eff }}<N_{\mathrm{T}}$ [31], where $N_{\mathrm{T}}=N_{\mathrm{p}} / 2$ is the adopted threshold (1ines 9-11).

\section{Case Study \& Evaluations}

Fig. 4 shows the parameters of the three-phase cross-linked polyethylene (XLPE) cable installation in trefoil formation analysed in this section. In order to validate the developed thermal model (cf. Subsection II-A), cable conductor temperature results in [16] have been used as a validation strategy.

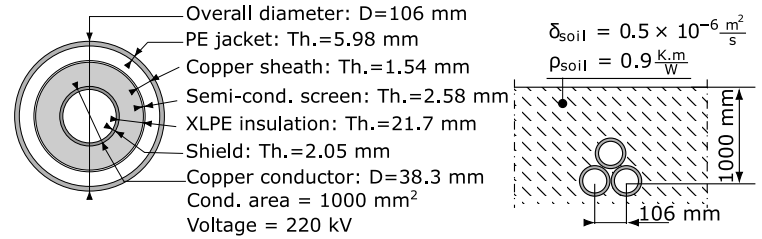

Fig. 4. Experimental case study parameters (Th: thickness, D: outer diameter).

Table I displays the lifetime estimation parameter values.

TABLE I

ARrheniUs IPM MOdEL PARAMETER VALUES [19].

\begin{tabular}{|c|c|}
\hline Parameter & Value \\
\hline$T T F_{0}[\mathrm{~h}]$ & $1 \times 10^{6}$ \\
\hline$b[\mathrm{~K} \mathrm{~mm} / \mathrm{kV}]$ & 4420 \\
\hline$\eta_{0}[$ non-dimensional $]$ & 15 \\
\hline$E_{0}[\mathrm{kV} / \mathrm{mm}]$ & 5 \\
\hline$E[\mathrm{kV} / \mathrm{mm}]$ & 7.2 \\
\hline$B[\mathrm{~K}]$ & 12430 \\
\hline
\end{tabular}

Cable layers, materials and their configuration in Fig. 4 influence the thermal model equations associated with cable losses, and in turn, the estimated cable conductor temperature directly influences the lifetime equation (see Subsection II-B).

\section{A. Diagnostics: cable health state estimation}

In order to calculate the health state under uncertainty, a number of load and ambient temperature profiles with different measurement errors have been examined. Fig. 5 shows the applied hourly sampled load and ambient temperature profiles. Note that the first 350 hour data samples of the load profile without errors are taken from [16], which is done deliberately to validate the obtained thermal results. This pattern is repeated periodically (see dashed vertical lines in Fig. 5) creating a signal of 14000 data samples.

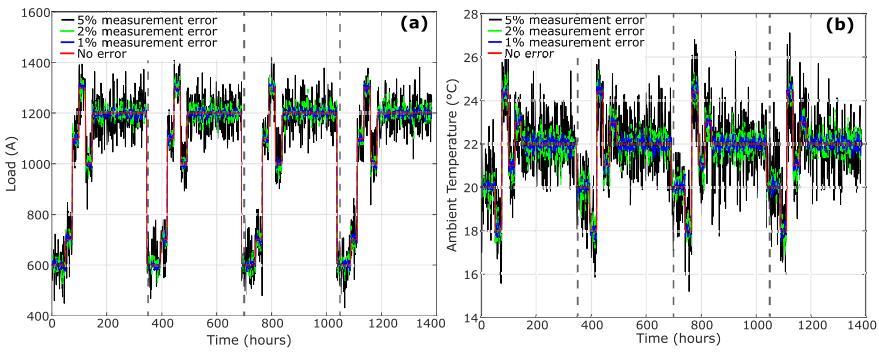

Fig. 5. (a) Load and (b) ambient temperature data samples and associated measurement errors (inspired from [16]).

For parametric sensitivity analysis purposes the influence of typical measurement errors of $1 \%, 2 \%$ and $5 \%$ have been tested for load $\psi_{I}=\{13 \mathrm{~A}, 26 \mathrm{~A}, 65 \mathrm{~A}\}$ and ambient temperature measurement errors $\psi_{a}=\left\{0.25^{\circ} \mathrm{C}, 0.5^{\circ} \mathrm{C}, 1.25^{\circ} \mathrm{C}\right\}$.
Under these assumptions, and considering a process measurement error of $\nu_{t}=0.0001$ units, an initial lifetime of $\nu_{R U L_{0}}=15$ years \pm 10 days $\left(\nu_{R U L_{0}}=\nu_{R U L_{t-1}}\right)$ and an initial cable temperature of $\Theta_{\text {cable }}(t=0)=20 \pm 0.5^{\circ} \mathrm{C}$, the associated lifetime reduction is calculated from the estimated cable conductor temperature [cf. Eq. (36)]. Adopted initial lifetime, process error and initial temperature values are hypothetical reasonable values and they can be adapted if needed.

Fig. 6a shows the cable conductor temperature over time for fixed 5\% ambient temperature measurement error and different load measurement errors. The maximum likelihood (ML) and 95\% confidence intervals (CI) are elicited from the pdfs inferred for each time instant using the PF model denoted as $\left[\mathrm{CI}^{-}, \mathrm{CI}^{+}\right]$. Fig. $6 \mathrm{~b}$ shows the pdf of the last cable conductor temperature data point, i.e. the 14000th sample in Fig. 6a. Fig. $6 c$ shows the cable lifetime reduction due to the thermal stress and Fig. 6d shows the pdf of the last lifetime estimate data point, i.e. 14000th sample in Fig. 6c.

Fig. 6a shows that the increase of load measurement error has a direct influence on the $95 \%$ confidence intervals of the cable conductor temperature. It can be noticed that the confidence bounds widen with the increased cable conductor temperature, which suggests that the measurement errors are more critical for higher cable conductor temperatures. Fig. $6 \mathrm{~b}$ shows the change of the pdf of the cable conductor temperature and it indicates that the greater the load measurement error, the wider the pdf bounds.

Fig. 6c shows that the greater the load measurement error, the lower the lifetime which is ruled according to the cable conductor temperature in Fig. 6a. The maximum likelihood values in Fig. 6c show a similar degradation trajectory for different load error values, but it can be noticed that the degradation for the highest load measurement error starts deviating faster towards the last 350 hours period due to higher cable conductor temperatures. Fig. 6d confirms that the maximum likelihood value is lower for greater load error values.

So as to evaluate the effect of cable conductor temperature estimates on the cable lifetime Fig. 7 shows the average life fraction lost per day, i.e. $F_{d a y}=1 / 24 \sum_{i=1}^{24} 1 / T T F_{i}$, which is repeated for the whole tested period. This index enables the observation of more stable trends.

It is possible to see from Fig. 7 that the effect of measurement errors on the loss-of-life fraction becomes more important over time as the cable conductor temperature increases. This can be seen with the difference between three loading cycles, were the confidence bounds of the $5 \%$ load error widen over the evaluation timespan.

\section{B. Sensitivity analysis}

Measurement errors influence directly on the cable conductor temperature, which in turn, directly impacts on the cable lifetime estimation. It is possible to correlate the cable ageing rate with the cable conductor temperature and its associated error for sensitivity analysis purposes.

In this context, the equivalent cable ageing (ECA) index as a function of the cable conductor temperature $\left(\Theta_{c}\right)$ and 


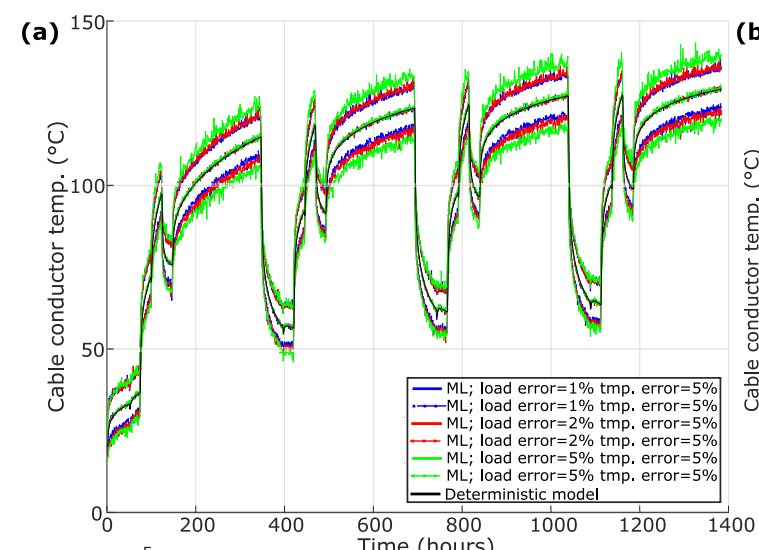

(b)
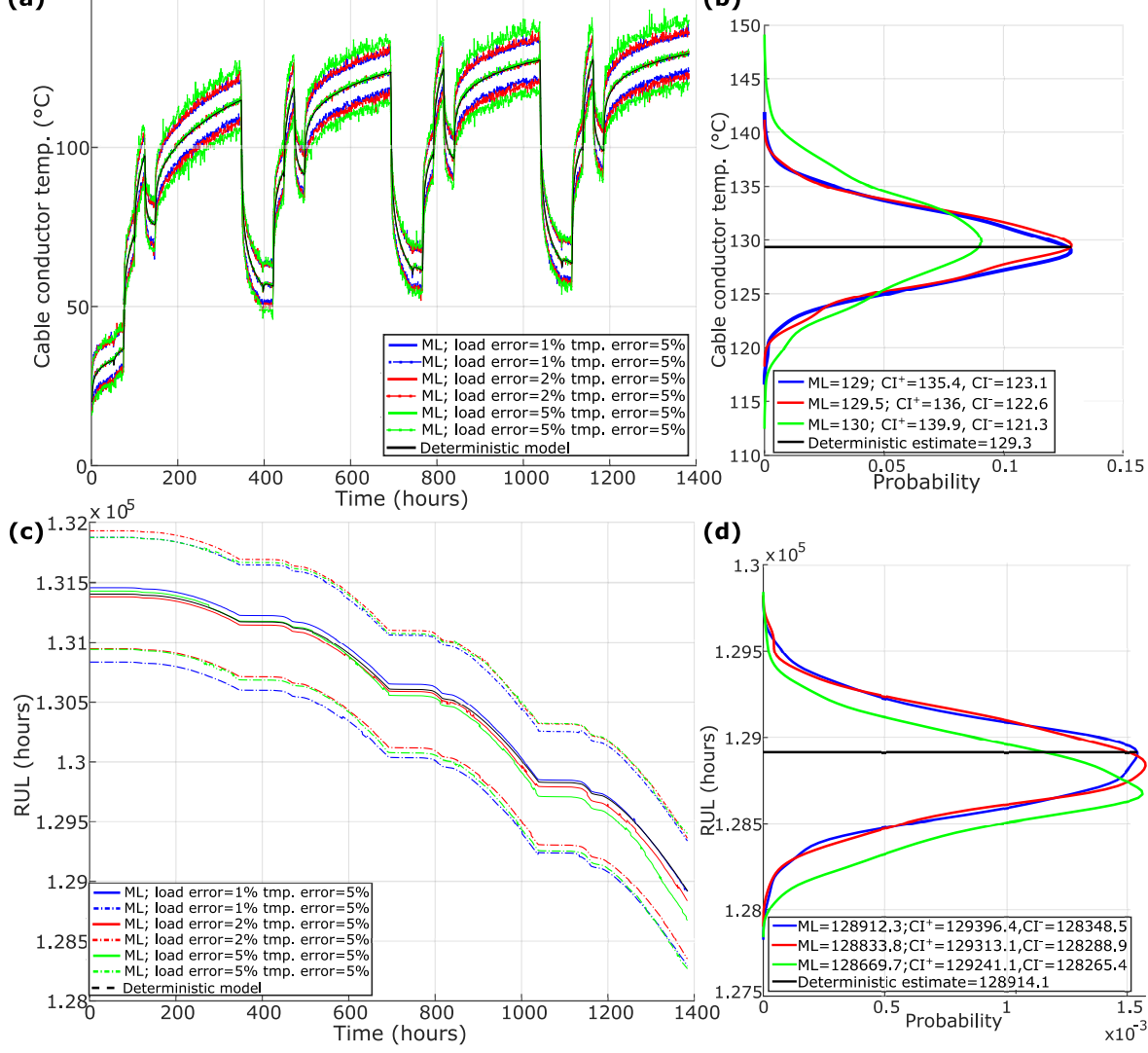

(d)

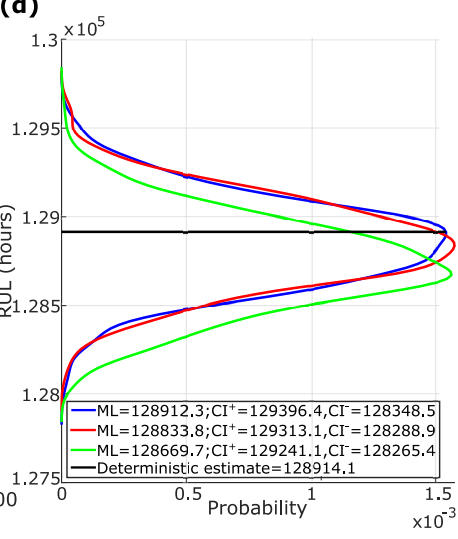

Fig. 6. (a) cable conductor temp.; (b) cable conductor temp. pdf at 14000th sample; (c) cable RUL estimate; (d) RUL pdf at 14000th data sample.

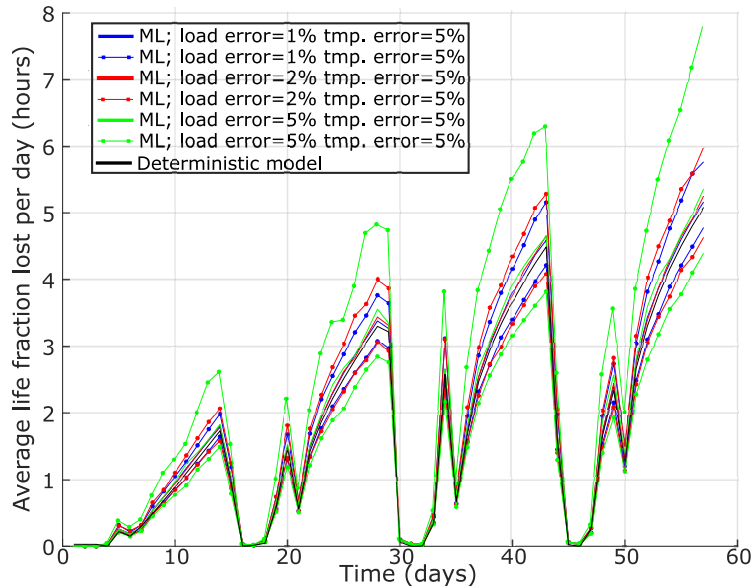

Fig. 7. Average life fraction lost per day.

its associated error $\left(e_{c}\right)$ can be defined by the ratio between the time-to-failure at the rated cable conductor temperature $\left(T T F_{R}\right)$ and the time-to-failure of the measured cable conductor temperature (TTF) [24]. The $E C A\left(\Theta_{c} \pm e_{c}\right)$ for a cable thermally rated at $90^{\circ} \mathrm{C}$ is defined as follows:

$$
E C A\left(\Theta_{c} \pm e_{c}\right)=\frac{T T F_{R}}{T T F}=e^{B\left(\frac{1}{363}-\frac{1}{\Theta_{c} \pm e_{c}}\right)} \frac{E}{E_{0}}-b\left(\frac{1}{366}-\frac{1}{\Theta_{c} \pm e_{c}}\right)
$$

Fig. 8 shows the ECA as a function of cable conductor temperature and associated cable conductor temperature errors.

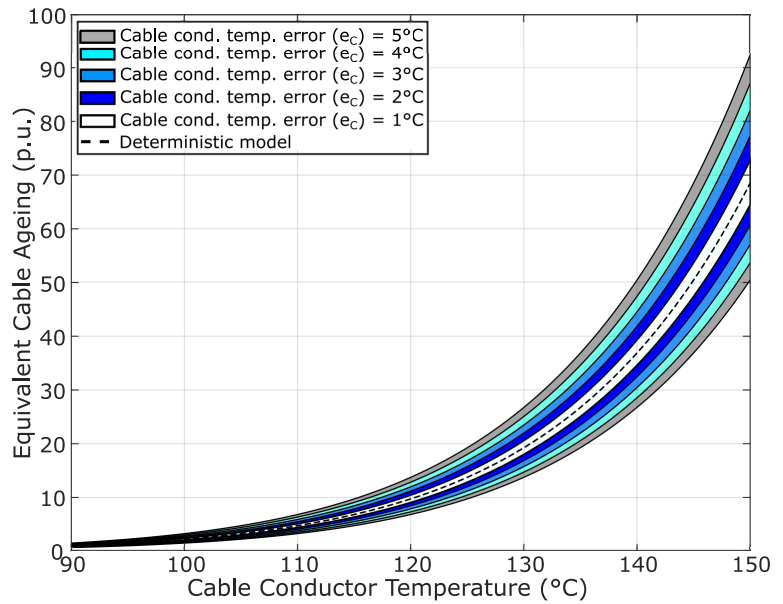

Fig. 8. Equivalent cable ageing for a cable thermally rated at $90^{\circ} \mathrm{C}$ as a function of cable temperature and error.

As Fig. 8 confirms the cable conductor temperature has a direct influence on the confidence intervals. Namely, the greater the cable conductor temperature the greater the influence of errors on the cable lifetime estimation. It can be noticed that the sensitivity of cable conductor temperature with respect to ECA is not lineal and this explains why the ageing rate is faster for different cable conductor temperatures and the effect of higher temperatures on the RUL estimate.

Note also that Fig. 8 informs about the equivalent cable ageing with respect to the rated cable. That is, at $90^{\circ} \mathrm{C}$ for the 
deterministic model the ECA is 1 unit, meaning that the ageing of the cable is equivalent to that of the rated temperature cable. However, this relationship changes quickly for different cable conductor temperature values.

The cable conductor temperature is influenced by different sources of uncertainty. Focusing on measurement uncertainties, the effect of load and ambient temperature measurement errors on cable lifetime can be analysed using the designed PF framework. Namely Fig. 9 shows the maximum likelihood and $95 \%$ confidence intervals of the last data point of the lifetime estimate using load and temperature profiles shown in Fig. 5. This is calculated for different load and ambient temperature measurement error values.

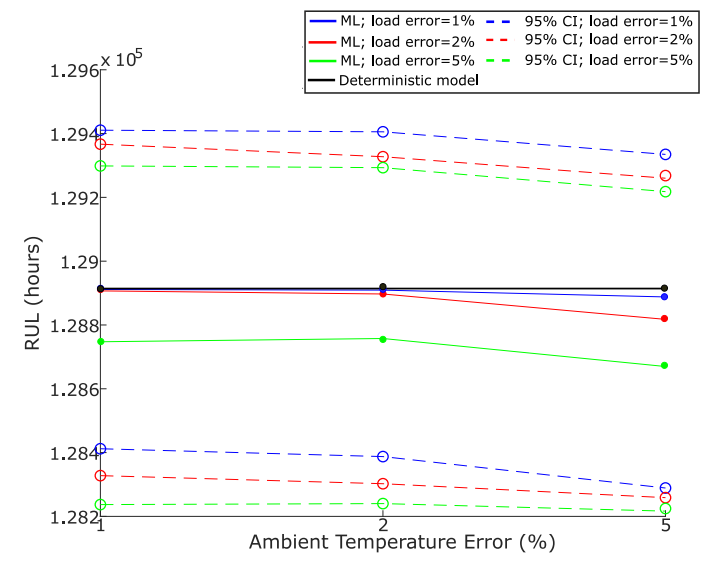

Fig. 9. Cable lifetime at 14000th data point for different error values.

It is possible to see from Fig. 9 that for the tested configurations the effect of load measurement error is greater than the ambient temperature measurement error. Namely, observing the maximum likelihood values, the lifetime variation of a fixed load error and different ambient temperature error values is lower compared with the lifetime variation of a fixed ambient temperature error and different load error values. It is also possible to see that the confidence bounds are greater for bigger measurement error values. The final shape of the PDF estimate depends on the uncertainty propagation mechanism of the Particle Filtering framework.

For all the evaluated metrics including cable conductor temperature, daily average life fraction lost, remaining useful life and equivalent cable ageing the corresponding probabilistic $95 \%$ confidence intervals have been estimated. These confidence bounds help the engineer in the decision-making process with information about the confidence of the estimate. Accordingly, this paper contributes with the integration of complex, detailed physics-of-failure operation and degradation process of cables including different sources of uncertainty within the Particle Filtering framework for probabilistic lifetime estimation purposes.

\section{Discussion}

There are different sources of uncertainty arising from the cable lifetime estimation process. The focus of this research is on measurement uncertainties (ambient temperature, load) and process uncertainties (degradation process, initial health state) because load and ambient temperature are measurable values widely used by practitioners, the uncertainty of the initial health state enables the adaptation of the framework to different health states and initial conditions, and the process uncertainty models the lack of exact knowledge of the empirical degradation modelling process. However, the designed diagnostics framework is extendible to other sources of uncertainty.

Focusing on the surrounding of the cable, it is possible to include other sources of uncertainty, such as soil or backfill, which are modelled with the equivalent thermal resistance $T_{4}$ in Fig. 1. In this work soil properties have been assumed constant, but it is possible to include soil-related uncertainties that impact on the cable conductor temperature and then integrate in the cable conductor temperature formulation. Refer to [16] [37] to see the effect of soil and backfill properties on the cable conductor temperature.

Similarly, the influence of different cable configurations on the lifetime estimate can be analysed through the appropriate adaptation of cable conductor temperature estimation equations. The cable arrangement affects the equations associated with the proximity and loss factors [cf. (7) and (11)], which in turn impact on the cable conductor temperature estimation.

If the type of cable changes including its constituent layers, this affects the equivalent electrical circuit in Fig. 1 and accordingly its associated thermal model formulations [15], [2].

These variations affect the cable conductor temperature estimation, $\Theta_{c}(\mathrm{t})$, which in turn affects the cable lifetime as examined in Fig. 8.

There are different cable configurations and sources of uncertainty that can be considered when estimating the cable conductor temperature and its lifetime. With the appropriate modifications of the underlying equations the proposed framework can accommodate different measurement and process uncertainties.

The specification of measurement and process errors is modelled using Gaussian pdfs and it is assumed that the distribution is centred on the measured value with the specified error values in Section IV (cf. Algorithm 2, line 5). However, note that the Particle Filtering framework is flexible and it enables the integration of non-Normal distributions too. In this direction, if the noise induced by measurement errors $\left(\psi_{I}\right.$, $\left.\psi_{a}\right)$ or process errors $\left(\nu_{R U} L_{0}, \nu_{t}\right)$ is known, it is possible to design the associated pdf from data which represents the corresponding measurement or cable health state process.

\section{CONCLUSIONS}

The effective development of power cable PHM strategies is challenging due to the involved complex degradation trajectories. The difficulty to obtain run-to-failure datasets has put the focus of many PHM applications on the development of physics-of-failure $(\mathrm{PoF})$ models. There are multiple sources of uncertainty that affect the specification of the PoF models. If these sources of uncertainty are not taken into account the decision-making process may not be accurate.

The literature indicates that an effective uncertainty processing framework for cable lifetime estimation is missing. 
In this direction, the diagnostics framework presented in this paper fills this gap with the proposed Bayesian state-estimation framework that connects data-driven probabilistic uncertainty models with PoF-based operation and degradation models. The framework systematically integrates different sources of uncertainty and propagates them to the final cable health state estimation for decision-making under uncertainty. This framework leads to a probabilistic decision-making process that impacts directly on the flexibility to adopt decisions. The evaluation of the cable health state under different conditions of uncertainty with associated confidence intervals assists in the decision-making under uncertainty.

The implemented sensitivity evaluation of the measurement errors improves the decision-making process with information about the effect of different sources of uncertainty on the cable lifetime. The analysis confirms that the influence of the ambient temperature measurement error on the lifetime estimate is greater than the load measurement errors. It has been also shown that the influence of load and ambient temperature measurement errors on the cable conductor temperature and lifetime estimates becomes more important for higher cable conductor temperatures.

\section{REFERENCES}

[1] C. Zhou, H. Yi, and X. Dong, "Review of recent research towards power cable life cycle management," High Voltage, vol. 2, no. 3, pp. 179-187, 2017.

[2] G. Anders, Rating of Electric Power Cables in Unfavorable Thermal Environment, ser. IEEE Press Series on Power Eng. Wiley, 2005.

[3] K. Anandakumaran, W. Seidl, and P. V. Castaldo, "Condition assessment of cable insulation systems in operating nuclear power plants," IEEE Trans. Dielectr. Electr. Insul., vol. 6, no. 3, pp. 376-384, Jun 1999.

[4] X. Peng, J. Wen, Z. Li, G. Yang, C. Zhou, A. Reid, D. M. Hepburn, M. D. Judd, and W. H. Siew, "Rough set theory applied to pattern recognition of partial discharge in noise affected cable data," IEEE Trans. Dielectr. Electr. Insul., vol. 24, no. 1, pp. 147-156, Feb 2017.

[5] C. K. Lee, G. Y. Kwon, S. J. Chang, M. K. Jung, J. B. Park, H. S. Kim, and Y. J. Shin, "Real-time condition monitoring of loca via timefrequency domain reflectometry," IEEE Transactions on Instrumentation and Measurement, vol. 66, no. 7, pp. 1864-1873, July 2017.

[6] T. Santhosh, V. Gopika, A. Ghosh, and B. Fernandes, "An approach for reliability prediction of instrumentation \& control cables by artificial neural networks and weibull theory for probabilistic safety assessment of npps," Reliability Eng. \& System Safety, vol. 170, pp. 31 - 44, 2018.

[7] G. Ye, H. Li, F. Lin, J. Tong, X. Wu, and Z. Huang, "Condition assessment of xlpe insulated cables based on polarization/depolarization current method," IEEE Transactions on Dielectrics and Electrical Insulation, vol. 23, no. 2, pp. 721-729, April 2016.

[8] S. Liu, Y. Wang, and F. Tian, "Prognosis of underground cable via online data-driven method with field data," IEEE Transactions on Industrial Electronics, vol. 62, no. 12, pp. 7786-7794, Dec 2015.

[9] T. Zhuang, M. Ren, X. Gao, M. Dong, W. Huang, and C. Zhang, "Insulation condition monitoring in distribution power grid via iot-based sensing network," IEEE Trans. Pow. Del., vol. 34, no. 4, pp. 1706-1714, 2019.

[10] Y. Huo, G. Prasad, L. Atanackovic, L. Lampe, and V. C. M. Leung, "Cable diagnostics with power line modems for smart grid monitoring," IEEE Access, vol. 7, pp. $60206-60220,2019$.

[11] F. Dinmohammadi, D. Flynn, C. Bailey, M. Pecht, C. Yin, P. Rajaguru, and V. Robu, "Predicting damage and life expectancy of subsea power cables in offshore renewable energy applications," IEEE Access, vol. 7, pp. 54658-54669, 2019.

[12] Z. Tang, W. Zhou, J. Zhao, D. Wang, L. Zhang, H. Liu, Y. Yang, and C. Zhou, "Comparison of the weibull and the crow-amsaa model in prediction of early cable joint failures," IEEE Transactions on Power Delivery, vol. 30, no. 6, pp. 2410-2418, Dec 2015.

[13] M. Buhari, V. Levi, and S. K. E. Awadallah, "Modelling of ageing distribution cable for replacement planning," IEEE Transactions on Power Systems, vol. 31, no. 5, pp. 3996-4004, Sept 2016.
[14] A. Said and R. A. Ghunem, "A techno-economic framework for replacing aged xlpe cables in the distribution network," IEEE Trans. Pow. Del., pp. $1-1,2020$

[15] IEC, "Calculation of the cyclic and emergency current rating of cables. part 2: Cyclic rating of cables greater than $8 / 30$ (36) kv and emergency ratings for cables of all voltages," IEC 60853-2008, 2008.

[16] M. Diaz-Aguilo and F. de Leon, "Adaptive soil model for real-time thermal rating of underground power cables," IET Science, Measurement Technology, vol. 9, no. 6, pp. 654-660, 2015.

[17] G. Mazzanti, "Analysis of the combined effects of load cycling, thermal transients, and electrothermal stress on life expectancy of high-voltage ac cables," IEEE Transactions on Power Delivery, vol. 22, no. 4, pp. 2000-2009, Oct 2007.

[18] F. De Leon and G. J. Anders, "Effects of backfilling on cable ampacity analyzed with the finite element method," IEEE Transactions on Power Delivery, vol. 23, no. 2, pp. 537-543, 2008.

[19] G. Mazzanti, "The combination of electro-thermal stress, load cycling and thermal transients and its effects on the life of high voltage ac cables," IEEE Transactions on Dielectrics and Electrical Insulation, vol. 16, no. 4, pp. 1168-1179, August 2009.

[20] Y. Bicen, "Trend adjusted lifetime monitoring of underground power cable," Electric Power Systems Research, vol. 143, pp. 189 - 196, 2017.

[21] R. Olsen, G. J. Anders, J. Holboell, and U. S. Gudmundsdóttir, "Modelling of dynamic transmission cable temperature considering soilspecific heat, thermal resistivity, and precipitation," IEEE Transactions on Power Delivery, vol. 28, no. 3, pp. 1909-1917, July 2013.

[22] E. Fernandez, I. Albizu, M. Bedialauneta, A. Mazon, and P. Leite, "Review of dynamic line rating systems for wind power integration," Renewable and Sustainable Energy Reviews, vol. 53, pp. 80 - 92, 2016.

[23] R. Dupin, G. Kariniotakis, and A. Michiorri, "Overhead lines dynamic line rating based on probabilistic day-ahead forecasting and risk assessment," International Journal of Electrical Power \& Energy Systems, vol. 110 , pp. $565-578,2019$.

[24] K. Kopsidas and S. Liu, "Risk-based cable circuit ratings for increasing wind power integration," IEEE Trans. Power Delivery, pp. 1-1, 2020.

[25] G. Mazzanti and G. C. Montanari, "A comparison between xlpe and epr as insulating materials for hv cables," IEEE Transactions on Power Delivery, vol. 12, no. 1, pp. 15-28, Jan 1997.

[26] S. Sankararaman, "Significance, interpretation, and quantification of uncertainty in prognostics and remaining useful life prediction," $M e$ chanical Systems \& Signal Processing, vol. 52-53, pp. 228 - 247, 2015.

[27] D. Villacci and A. Vaccaro, "Transient tolerance analysis of power cables thermal dynamic by interval mathematic," Electric Power Systems Research, vol. 77, no. 3, pp. 308 - 314, 2007.

[28] C. Wang and Z. Qiu, "Subinterval perturbation methods for uncertain temperature field prediction with large fuzzy parameters," International Journal of Thermal Sciences, vol. 100, pp. 381 - 390, 2016.

[29] H. Shabani and B. Vahidi, "A probabilistic approach for optimal power cable ampacity computation by considering uncertainty of parameters and economic constraints," International Journal of Electrical Power \& Energy Systems, vol. 106, pp. 432 - 443, 2019.

[30] R. Billinton and D. Huang, "Effects of load forecast uncertainty on bulk electric system reliability evaluation," IEEE Transactions on Power Systems, vol. 23, no. 2, pp. 418-425, May 2008.

[31] M. S. Arulampalam, S. Maskell, N. Gordon, and T. Clapp, "A tutorial on particle filters for online nonlinear/non-gaussian bayesian tracking," IEEE Trans. Signal Processing, vol. 50, no. 2, pp. 174-188, Feb 2002.

[32] G. Yilmaz and S. E. Karlik, "A distributed optical fiber sensor for temperature detection in power cables," Sensors and Actuators A: Physical, vol. 125, no. 2, pp. 148 - 155, 2006.

[33] IEC, "Electric cables - calculation of the current rating: Current rating equations (100\% load factor) and calculation of losses - general," IEC Standard 60287-1-1, 2006.

[34] J. Teh, C. Lai, and Y. Cheng, "Impact of the real-time thermal loading on the bulk electric system reliability," IEEE Transactions on Reliability, vol. 66, no. 4, pp. 1110-1119, Dec 2017.

[35] G. Mazzanti, "The effects of seasonal factors on life and reliability of high voltage ac cables subjected to load cycles," IEEE Transactions on Power Delivery, 2019.

[36] J. Aizpurua, S. McArthur, B. Stewart, B. Lambert, J. Cross, and V. Catterson, "Adaptive power transformer lifetime predictions through machine learning and uncertainty modeling in nuclear power plants," IEEE Trans. Ind. Electronics, vol. 66, no. 6, pp. 4726-4737, 2019.

[37] F. de Leon and G. J. Anders, "Effects of backfilling on cable ampacity analyzed with the finite element method," IEEE Transactions on Power Delivery, vol. 23, no. 2, pp. 537-543, 2008. 